\title{
Phosphatidylethanol (PEth); the Superior Direct Alcohol Biomarker against Mean Corpuscular Volume (MCV), Aspartate Amino Transferase (AST), Alanine Aminotransferase (ALT), Gamma-Glutamyl Transferase (GGT) and Carbohydrate-Deficient Transferrin (CDT)
}

\author{
Salah Eddine Breidi* \\ Head of Forensic Toxicology, DNA Legal, Frome, United Kingdom \\ *Corresponding author: Salah Eddine Breidi, Head of Forensic Toxicology, DNA Legal, Frome, United Kingdom \\ To Cite This Article: Salah Eddine Breidi. Phosphatidylethanol (PEth); the Superior Direct Alcohol Biomarker against Mean Corpuscular \\ Volume (MCV), Aspartate Amino Transferase (AST), Alanine Aminotransferase (ALT), Gamma-Glutamyl Transferase (GGT) and Carbohydrate- \\ Deficient Transferrin (CDT). Am J Biomed Sci \& Res. 2019 - 3(1). AJBSR.MS.ID.000623. DOI: 10.34297/AJBSR.2019.03.000623
}

Received: May 02, 2019 | Published: May 10, 2019

\section{Editorial}

As ethanol itself is metabolised and excreted quickly from the body, assessing alcohol consumption with a blood test relies on other markers indicative of consumption. Traditional tests include Liver Function Testing (LFT), Carbohydrate Deficient Transferrin (CDT) Testing, and a Full Blood Count (FBC). Phosphatidylethanol (PEth) testing, on the other hand, is a direct biomarker of alcohol consumption, can only be detected when alcohol has been consumed, and is directly correlated with the level of alcohol consumed

There are some difficulties when testing alcohol in blood to determine if alcohol had been abused. Due to these challenges in testing alcohol consumption behaviours across society, in the most recent decades, intensive research has been conducted to find reliable biomarkers that are efficient enough to discriminate chronic excessive drinking. Initially, the markers are divided into two categories direct and indirect alcohol biomarkers. Indirect biomarkers, which is a secondary biomarker, detect the effects of alcohol on organ systems by measuring the elevated levels of the body chemistry after alcohol consumption. The most commonly used indirect biomarkers are mean corpuscular volume (MCV), aspartate aminotransferase (AST), alanine aminotransferase (ALT), gamma-glutamyl transferase (GGT) and carbohydrate-deficient transferrin (CDT).

With the indirect biomarkers, more than one factor (including alcohol) can lead to an elevation in the measured level in blood. Smoking and several Pharmaceutical drugs may increase (induce)

the concentration of GGT in the blood. In addition, chronic disease or Vitamin B12/folate deficiencies can increase the mean corpuscular volume (MCV). CDT can also be affected by factors such as hormonal changes and other diseases and deficiencies [1].

Phosphatidylethanol (PEth) is a direct biomarker. The test is not a new method for monitoring alcohol consumption. There are a number of old and new studies that proved the superiority of PEth test over the other indirect blood alcohol markers. PEth exhibits high diagnostic sensitivity and specificity for detecting active chronic excessive drinking behaviours. Phosphatidylethanol (PEth) is produced in the presence of low quantities of alcohol [1]. On average, PEth can be detected for nearly 28 days depending on the consumed amounts [2-5].

Previous experiments and tests were conducted using less sensitive HPLC methods. Recent drinking experiments, employed a more sensitive LC-MS/MS method for the quantification of PEth showed that the formation of PEth began immediately after the volunteers started drinking alcohol (1 hour), reaching a concentration of about $35-237 \mu \mathrm{g} / \mathrm{L}$. The measured levels of PEth increased continuously over the next days and reached the maximum concentrations between days 3 and 6 [1,6-9].

The previously mentioned indirect biomarker are insensitive when tested separately and also incapable to determine abstinence. In 2016, DNA legal is one of the rare laboratories around the United Kingdom to develop a method that is capable of identifying abstinence amongst the tested individuals. 
What is concluded from this review, PEth detection period is up to 3-4 weeks. PEth is a direct biomarker that is capable to differentiate between teetotallers, social, chronic and excessive drinking behaviours [10,11]. PEth values can be translated into an approximate level of alcohol consumption. PEth appears to be a more reliable measure of alcohol consumption than self-reports".

Recent research in 2013 by Skipper GE et al. [10] was clear on the advantage of PEth ability in combination with previous low positive EtG results to differentiate between innocent/extraneous exposure and drinking. The same research stated that blood PEth is only positive following significant alcohol use which makes it a good alcohol marker [12]. Another recent study supported Peth test being a reliable biomarker of alcohol consumption when LFT results are incapable to determine the alcohol consumption in individuals with damaged liver [13].

Other published studies in 2014 and 2015 presented the specificity of PEth as a direct biomarker and the ability to monitor social and excessive drinking habits $[14,15]$. The study by Walther L et al. [16] also introduced the superiority of PEth over the other alcohol biomarkers by comparing the correlation of self-reported results of PEth and the other biomarkers. The author concluded that "At all consumption levels, PEth was superior to CDT owing to its substantially higher sensitivity and also due to its closer correlation to self-reported alcohol consumption". The present studies demonstrate a good clinical efficiency "up to $99 \%$ sensitivity" of PEth for detecting alcohol drinking [8,14-16] (Table 1).

\begin{tabular}{|c|c|}
\hline Table 1: Recommended cut off level. & What does it mean? \\
\hline If & Abstinent or very low consumption in the past 28 days \\
\hline PEth: $<10 \mu \mathrm{g} / \mathrm{L}$ & Low/occasional alcohol consumption in the past 28 days $10-35 \mu \mathrm{g} / \mathrm{L}$ \\
\hline PEth: $35-210 \mu \mathrm{g} / \mathrm{L}$ & Social/Moderate alcohol consumption in the past 28 days \\
\hline PEth: $>210 \mu \mathrm{g} / \mathrm{L}$ & Excessive alcohol consumption in the past 28 days \\
\hline
\end{tabular}

*When compared to other PEth tests performed by the laboratory (DNA Legal, 2019).

\section{References}

1. Viel G, Boscolo-Berto R, Cecchetto G, Fais P, Nalesso A, et al. (2012) Phosphatidylethanol in Blood as a Marker of Chronic Alcohol Use: A Systematic Review and Meta-Analysis. Int J Mol Sci 13(11): 1478814812 .

2. Aradottir S, Asanovska G, Gjerss S, Hansson P, Alling C (2006) Phosphatidylethanol (PEth) concentrations in blood are correlated to reported alcohol intake in alcohol-dependent patients. Alcohol and alcoholism 41(4): 431-437.

3. Varga A, Hansson P, Johnson G (2000) Normalization rate and cellular localization of phosphatidylethanol in whole blood from chronic alcoholics. Clinica Chimica Acta 299(1-2): 141-150.

4. Orrego H, Blake JE, Israel Y (1985) Relationship between gammaglutamyl transpeptidase and mean urinary alcohol levels in alcoholics while drinking and after alcohol withdrawal. Alcohol Clin Exp Res 9(1): 10-13.

5. (2016) The Use of Phosphatidylethanol as a Direct Alcohol Biomarker: An Annotated Bibliography, United States Drug Testing Laboratories, USA.

6. Gnann H, Weinmann W, Thierauf A (2012) Formation of phosphatidylethanol and its subsequent elimination during an extensive drinking experiment over 5 days. Alcohol Clin Exp Res 36(9): 15071511.

7. Steina Aradóttir, Kristian Moller, Christer Alling (2004) Phosphatidylethanol formation and degradation in human and rat blood. Alcohol and Alcoholism 39(1): 8-13.

8. Helander A, Peter O, Zheng Y (2012) Monitoring of the alcohol biomarkers PEth, CDT and EtG/EtS in an outpatient treatment setting Alcohol Alcohol 47: 552-557.
9. United States Drug Testing Laboratories, PEth testing, $7^{\text {th }}$ April 2016.

10. Skipper GE, Thon N, Dupont RL, Baxter L, Wurst FM (2013) Phosphatidylethanol: The potential role in further evaluating low positive urinaryethyl glucuronide and ethyl sulfate results. Alcohol Clin Exp Res 37: 1582-1586.

11. Marques P, Hansson T, IsakssonA (2011)Detection of phosphatidylethanol (PEth) in the blood of drivers in an alcohol ignition interlock program. Traffic Inj Prev 12(2): 136-141.

12. Skipper GE, Thon N, Dupont RL, Baxter L, Wurst FM (2013) Phosphatidylethanol: The Potential Role in Further Evaluating Low Positive Urinary Ethyl Glucuronide and Ethyl Sulfate Results, Alcoholism: Clinical and Experimental Research 37(9): 1582-1586.

13. Stewart SH, Koch DG, Willner IR, Anton RF, Reuben A (2014) Validation of Blood Phosphatidylethanol as an Alcohol Consumption Biomarker in Patients with Chronic Liver Disease. Alcohol Clin Exp Res 38(6): 17061711.

14. Schröck A, Hernández RA, Martin FM, König S, Weinmann W (2015) 'Phosphatidylethanol (PEth) in blood samples from "driving under the influence" cases as indicator for prolonged excessive alcohol consumption. International Journal of Legal Medicine 130(2): 393-400.

15. Jennifer Jain, Jennifer L Evans, Alya Briceño, Kimberly Page, Judith A Hahn (2014) Comparison of Phosphatidylethanol Results to SelfReported Alcohol Consumption Among Young Injection Drug Users. Alcohol and Alcoholism 49(5): 520-524.

16. Walther L, de Bejczy A, Löf E, Hansson T, Andersson A (2015) Phosphatidylethanol is Superior to Carbohydrate-Deficient Transferrin and $\gamma$-Glutamyltransferase as an Alcohol Marker and is a Reliable Estimate of Alcohol Consumption Level. Alcohol Clin Exp Res 39(11): 2200-2208. 